\title{
The Discussion of 500kV Centralized Monitoring System for Large Operation and Large Maintenance Mode
}

\author{
Shuai-lingling, Liu-Xiaojiang, Li-Jun \\ Jiangxi Electric Power Co. Maintenance Branch, Nanchang, China \\ Email: abcshuai@163.com
}

Received 2013

\begin{abstract}
The establishment of a centralized monitoring system is an effective means to achieve the goal that no or fewer people are on duty. The function of the current $500 \mathrm{kV}$ centralized monitoring system is not enough mature to meet the requirement of the large-scale operation and large-scale maintenance model proposed by the State Grid. In order to fully utilizing the function of the centralized monitoring system, an improved design scheme is presented for the current $500 \mathrm{kV}$ centralized monitoring of the Jiangxi Power Grid. The improved centralized monitoring system is divided into three layers: monitoring center layer, operating team layer, and substation layer, which not only has the advantages of high security, high reliability, and easy maintenance, but also realizes the management model of regional control. So it can perfectly meet anti-misoperation requirement in the large operation and large maintenance mode, which can provide reference and guidance for the design of the $500 \mathrm{kV}$ centralized monitoring system of Jiangxi Power Grid.
\end{abstract}

Keywords: Centralized monitoring system; Substation; Anti-misoperation system; Five-prevention system

\section{Introduction}

With the rapid development of computer technology, communication technology, and automatic control technology, the operating mechanism for no or fewer people on duty is a development trend of the substation[1-2], and the establishment of a centralized control system is an effective means to achieve the goal. Currently, many power companies have established the centralized control system to achieve the management model of the centralized control, unified dispatching, and unified maintenance for all substations[3-5]. This not only meets the requirements of the rapid development of the State Grid, but also conforms the requirements of the intensified management of three components and integrated systems of five processes proposed by the State Grid Corporation.

However, the function of $500 \mathrm{kV}$ centralized monitoring system of Jiangxi Power Grid is still not enough mature, which exists some problems and shortcomings. In order to adapt the development requirements of the large-scale operation and large-scale maintenance, the power company intends to reform the current system. Therefore, this paper analyzes the problems of the current system. Based on that, a new design scheme is proposed, and the new centralized monitoring system is divided into three layers: monitoring center layer, operating team layer, and substation layer. The improved de- sign can meet the anti-misoperation requirement of the large-scale operation and large-scale maintenance model, which can provide reference and guidance for the design of $500 \mathrm{kV}$ centralized monitoring system of Jiangxi Power Grid.

\section{The Problems of the Current Centralized Monitoring System}

\subsection{The Present Situation}

There are $15500 \mathrm{kV}$-substations in Jiangxi. The $500 \mathrm{kV}$ monitoring center mainly monitors the operation conditions of the $15500 \mathrm{kV}$-substations, and the structure diagram is shown in Figure 1.

This system achieve the "four remote" function, which can monitor the operation situation of every substation via Ethernet. The attendants in the substation mainly response to the equipment operation and switch operation according to the instruction of the dispatching center. Firstly, the attendants accept the instructions of the dispatching center and make an operation order. Secondly, the switching simulation is performed in the five-prevention workstation of the substation, and the operation order is downloaded to the computer key after the success of the switching simulation. At last, the switching operation of the equipment is performed uti- 
lizing the computer key, and the operation order is returned to the five-prevention workstation after completed.

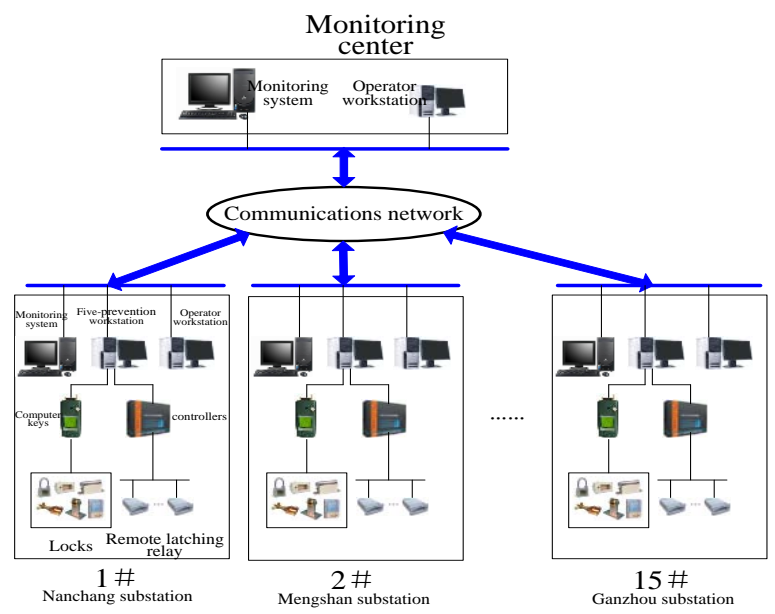

Figure 1. The structure diagram of the current $500 \mathrm{kV}$ centralized monitoring system of Jiangxi Power Grid

\subsection{The Current Problems}

According to the development requirements of large-scale operation and large-scale maintenance model proposed by the State Grid, some problems still exist in the centralized monitoring system. The main problems specialize in the following five aspects: (1) The centralized monitoring system is composed of the monitoring center layer and substation layer, which can not achieve the regional control.(2) Some people must be on duty in each substation, and the numbers of the attendants are more than 15, which need a lot of attendants. (3) The monitoring center has the ability of the remote operation function for the electrical equipment in the substation. However, the monitoring center doesn't exert the remote operation function in the actual operation process in case of the design faults of the centralized monitoring system. (4) The monitoring center has not five-prevention server and five-prevention workstation, which is difficult to manage the operation data of all of substations, and can not make the operation order. (5) The master video monitoring system in the monitoring center only inspects all of substation and is not collected to the master monitoring system, so its anti-misoperation function is fully utilized.

\section{The New Requirements of Anti- misoperation for the Centralized Monitoring System}

The operation manage model of the current $500 \mathrm{kV}$ centralized monitoring system of Jiangxi Power Grid is the two layers model concluding the monitoring center layer and substation layer, which can not meet the anti- misoperation requirement of the large-scale operation and large-scale maintenance model.

(1) In the construct system of large-scale operation and large-scale maintenance model, the ways of the electrical equipment operation increase, for example remote operation, local operation and maintenance operation, and the operation places increase accordingly including monitoring center, observation panel, terminal box and the local operation mechanisms. Therefore, the anti-misoperation system of the centralized monitoring system should be layered, and each layer should be independent and communicated each other.

(2) Currently, some important anti-misoperation information of the substation does not upload to the monitoring center, for example, the temporary ground line, which directly influences the security of the remote operation in the monitoring center.

(3) Currently, the microcomputer anti-misoperation system in the substation only achieves the anti-mi- soperation function of local substation, and is lack in that of the regional power grid. So the centralized monitoring system should possess the locking function for the equipment of collected lines between substations.

In addition, the centralized monitoring system should have management function of the unique operation right and the maintenance anti-misoperation function for the electrical equipments.

\section{The Design of the Centralized Monitoring System}

\subsection{The Design Target}

The centralized monitoring system should be provided with the function of centralized control, unified dispatching, and unified maintenance for all substations. So, the system must reflect the operation conditions of each substation accurately and real-time.

(1) High security

In order to avoid the power system accidents caused by misoperation, the multi-layer anti-misoperation system which focuses on the five-prevention server in the monitoring center is built to make the operation of the power system safe and stable.

(2) High reliability

Five-prevention server, the most critical component of the system, is responsible for the centralized management for the all substations, the remote operation in the monitoring center layer and the management of the unique operation right.

In addition, the communication between the operation data of all substations and five-prevention server, 
and the communication between the layers of the monitoring system are realized via network. Therefore, the centralized monitoring system should adopt the redundancy mechanism of double-server and double-channel to ensure the reliable operation of the power system.

\section{(3) Convenient maintenance}

Data maintenance of the centralized monitoring system can be performed not only in the monitoring center, but also in the five-prevention station or in the substation. The data automatically synchronized after completed.

\section{(4) Less investment, easy expansion}

Multi-layer anti-misoperation locking function of the centralized monitoring system should be built on the existing anti-misoperation system to avoid repeat investment. At the same time, the construction of the centralized control system should be easy to access the new substation in the future.

\subsection{The Structure Diagram of the Centralized Monitoring System}

In order to solve the existing problems of $500 \mathrm{kV}$ centralized monitoring system in Jiangxi Power Grid and to meet the development requirements of the large-scale operation and large-scale maintenance, the current centralized monitoring system is improved and divided into three layers: monitoring center layer, operating team layer and substation layer. Every layer connects each other through network. The structure diagram of centralized monitoring system is shown in Figure 2.

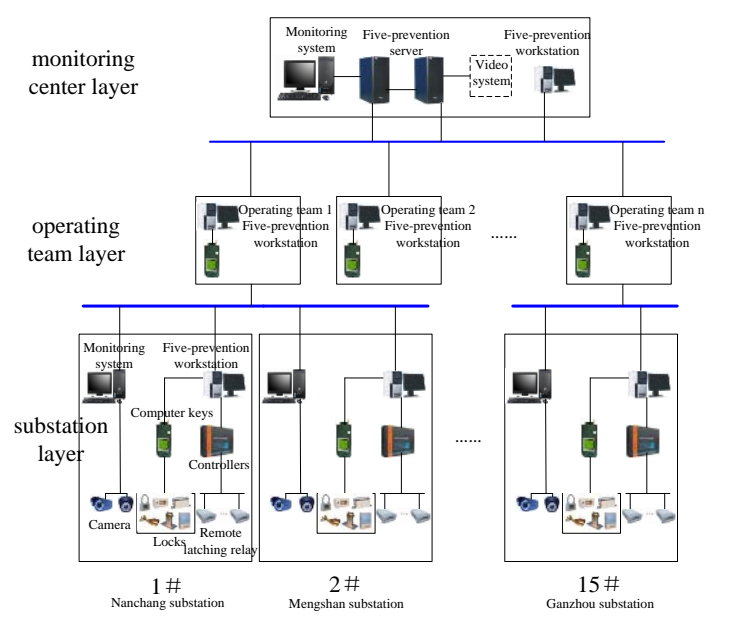

Figure 2. The structure diagram of the improved $500 \mathrm{kV}$ centralized monitoring system

As shown in Figure 2, the centralized monitoring system takes the monitoring center layer as the core and controls the other layers. The monitoring center layer is configured with five-prevention server which stores the real-time data information of all substations. The server can complete the functions including centralized management, statistics, and inquiry for the substations' data. Meanwhile, it has the anti-misoperation function including making operation order, switching simulation, locking remote operation in the monitoring center layer, and the unique operation right for the electrical equipment. At the same time, the server has dual-redundant function. When the host server fails, the backup server automatically operates to improve the reliability of the power system. In addition, the monitoring center also establishes five-prevention workstation, which can complete anti-misoperation switching simulation and send operation order for every substation controlled by the centralized monitoring system.

Five-prevention workstation of the operating team layer uses the computer as the operation interface, which can display the equipment situation and operating model of the controlled substation. At the same time, five-prevention workstation has the functions of making operation order and switching simulation.

The five-prevention workstation of the substation is governed by the five-prevention server. With the growing number of substations, it can implement the management distribution among the layers by adjusting the permission. The substation can be configured with independent five-prevention system to implement anti-misoperation locking function for equipments operation.

\subsection{The Settings of the Operating Team}

In order to achieve regional control for the Jiangxi $500 \mathrm{kV}$ substations, four operating teams will be established according to the geographic distribution of the substations. The principle of building operating team is the closer distance between one substation where the operating team situated and the other substation which the operating team need to maintenance. The distribution of operating team is shown in Table 1.

Table 1: Distribution of the operating team

\begin{tabular}{ccc}
\hline NO. & Place & Station name \\
\hline & & Mengshan \\
Nanchang \\
Operating \\
team I & Monitoring center & $\begin{array}{c}\text { Yongxiu } \\
\text { Jingxian } \\
\end{array}$ \\
& & Shizhongshan \\
& & Fuzhou \\
Operating & Yingtan ubstation & Hongyuan \\
team II & & Leping \\
& & Yingtan \\
Operating & Luofang substation & Luofang \\
team III & & Anxiang \\
Operating & Ganzhou substation & Wenshan \\
& & Leigongshan
\end{tabular}




\subsection{The Anti-misoperation Function of the Centralized Monitoring System}

(1) Anti-misoperation criterion of the centralized monitoring system needs not only the switch signal of the electrical equipment, but also the electrical remote measurement signal(such as voltage, current). For achieving real-time alignment function in the whole operation process, it needs to check not only the state of the electrical equipment before operating, but also the state of the electrical equipment in the process of the operation.

(2) Centralized monitoring system has perfect remote operation locking function, that the monitoring center and monitoring system in the substation can realize remote operation for each substation by the soft locking (communication) and hard locking (remote control circuit hard contacts).

(3) The centralized monitoring system and the video system connect together. The staffs in the monitoring center can not only real-time monitor the substation operator on-site operation, such as verify the operating conditions of the electrical equipment, but also inspect the surrounding environment of the substation.

\subsection{The Operation Model of the Centralized Monitoring System}

The centralized monitoring system has many kinds of operation models.

Operation model 1: The attendant in the monitoring center makes an operation order in accordance with the command of the dispatching center, and the switching simulation is performed in the five-prevention workstation of the monitoring center. The operation order is sent to the corresponding operating team via network after succeed and transfer to computer key, and the operators of the operating team take computer key and carry out the switching operation. The operation order is returned to the five-prevention server in the operating team layer or the substation layer to ensure the consistency of device status information after completed.

Operation model 2: The attendant in the operating team layer makes an operation order in accordance with the command of the dispatching center, and the switching simulation is performed in the five-prevention workstation of the operating team. The operation order is transferred to computer key after succeed, and the operators of the operating team take computer key and carry out the switching operation. The operation order is returned to the five-prevention server in the operating team layer or the substation layer to ensure the consistency of device status information after completed.
Operation model 3: The attendant in the operating team layer makes an operation order in accordance with the command of the dispatching center, and the switching simulation is performed in the five-prevention workstation of the operating team. The operation order is sent to the corresponding substation via network after succeed and transfer to computer key, and the operators of the substation take computer key and carry out the switching operation. The operation order is returned to the five-prevention server in the substation layer to ensure the consistency of device status information after completed.

Operation model 4: The attendant in the substation layer makes an operation order in accordance with the command of the dispatching center, and the switching simulation is performed in the five-prevention workstation. The operation order is transferred to computer key after succeed, and the operators of the substation take computer key and carry out the switching operation. The operation order is returned to the five-prevention server in the substation layer to ensure the consistency of device status information after completed.

The centralized monitoring system has clear structure, which solves the existing problems effectively. It possesses anti-misoperation function for the electrical equipment operation perfectly from many aspects: right-management, the sole operating rights management, switching simulation, the real-time logic judgment, and locking equipment. The system has the advantages of high security, high reliability, and easy maintenance, which can absolutely meet the new requirements of the anti-misoperation system for the large-scale operation and large-scale maintenance model.

\section{Conclusions}

According to the development requirements of the largescale operation and large-scale model proposed by the State Grid, the existing problems of the current $500 \mathrm{kV}$ centralized monitoring system of Jiangxi Power Grid are analyzed. It transforms the two-layer management model of the monitoring center layer and substation layer of current $500 \mathrm{kv}$ centralized monitoring system to the three-layer management model, that is monitoring center layer, operating team layer and substation layer. Besides, the functions of the improved centralized monitoring system are elaborated. The system possesses the advantages of high security, high reliability and easy maintenance, which can meet the new requirements of the anti-misoperation system for the large-scale operation and large-scale maintenance model and provide reference for the design of the centralized monitoring system. 


\section{REFERENCES}

[1] ZHAO Jiaqing. Computer centralized SCADA system on unmanned $220 \mathrm{kV}$ substation. Automation of Electric Power Systems, Vol.27, No.8, 2003, pp. 78-80.

[2] LIANG Chao-mei ,CHEN Jian-feng.The Implementation of Unmanned Substation Automation. Electric Power Automation Equipment, Vol. 20, No. 4, 2000, pp. 63-65.

[3] LIANG Yun-hua, SHEN Meng-tian. Realization of real-time remote maintenance in $500 \mathrm{kV}$ substation automation system. Power System Protection and Control,
Vol. 38, No. 14, 2010, pp.165-168.

[4] ZHU Dongsheng, SUN Chunjun, CHEN Fei. Centralized remote monitoring system of $500 \mathrm{kV}$ substation[J]. Electric Power Automation Equipment, Vol. 29, No. 5, 2009, pp. 126-129.

[5] GU Wenqi, MO Jie, HE Yanying. Centralized control type anti-misoperation operating system design. Electric Power Automation Equipment, Vol. 30, No. 7, 2010, pp. 127-130. 